\title{
Forecast on Short-Term Wind Speed and Wind Farm Power Generation Yiping Cheng ${ }^{1}$ \\ ${ }^{1}$ North China Electric Power University, Beijing, China 102206 \\ hunter2011@foxmail.com
}

Keywords: Wind Power; Wind Speed; Forecast

\begin{abstract}
Wind forecast is of great significance for operating a wind farm and power systems. The accurately predict wind speed can effectively reduce the adverse impact of wind farms on the power system, while increase the wind farm in the electricity market competitiveness. This paper studied time series and neural network forecasting for wind speed, and as a basis for proposed change right combination of multi-model prediction of wind speed forecasting. This method takes into account the characteristics of each individual prediction methods, different combinations of individual prediction methods to improve the prediction accuracy. Finally, wind power predicted by the results of comparative analysis found that for the prediction accuracy of wind speed is higher than the wind power prediction accuracy.
\end{abstract}

\section{Introduction}

Renewable energy, especially wind energy development and utilization has been attached great importance to the world [1]. Wind power is an environmentally friendly, renewable energy, as the next major forms of energy, its human way of life, survival and development are of great significance in the future. Wind speed and wind power forecasting is a prerequisite for large-scale wind farms and network operation, and more accurate forecasts in favor of the timely adjustment of power system dispatch department scheduling; can reduce the impact of wind power on the grid is unstable; you can also participate in the auction for the generation of wind farms foundation.

Short-term wind power prediction refers forecast within the next 72 hours. The most commonly used method of generating short-term prediction of wind power are mainly time series, support vector machines, neural network method and signal decomposition method, each forecasting method has its advantages, but there were also some errors. Taking Zhangjiakou Wind Farm, for example, for different kinds of prediction methods were studied. Articles were used time series method and neural network modeling, and based on this paper, a combination of multi-model prediction method, and then obtaining the prediction error calculated by examples of different approaches, and thus the three prediction methods were compared and Analysis.

\section{Wind Power Generation Characteristics}

Features of Wind Power. Wind farm is achieved through wind turbines wind power, wind turbine wind wheel is the most important part. When the wind speed ranged between cut-in speed and cut-out wind speed, turbine output is proportional to the cube of the wind velocity relationship. This shows that the fan output power and wind speed have a great relationship.

Its primary energy by wind power - Wind energy limitations, there are some of the following features. (1) The wind is very unstable. (2) Wind energy storage difficulties. (3) The geographic distribution of wind farms more remote. Advantages (4) environmental benefits of wind power is good, it does not emit any polluting gases and waste.

Fan Speed-power Relations. Wind farm wind power output fluctuation and volatility. In unit time vertical flow through cross-sectional area of wind energy $P=\frac{1}{2} C A v^{3}$, namely wind turbine output power. But in practice, wind energy has not all been absorbed by the turbine, so fans can catch wind power represented by the following formula: 


$$
P=\frac{1}{2} C_{p} A \rho v^{3}
$$

Where, $P$ for the fan output; the wind swept round the area $A=\pi R^{2}$; for the wind wheel radius $R$; the air density $\rho$; wind speed $v$; wind energy utilization coefficient fans $C_{p}$, a maximum value of 0.593 .

\section{Wind Speed Forecast of ARMA Model Based On Time Series}

Fundamental. SCADA certain time intervals can capture and record the average output power of each fan, these observations ordered random data to form a power time series. Time series contains all the information generated historical behavior of the sequence of a system, to analyze its current and prior observations finite length, establish the corresponding parameters of the model, and then use this model in the future to predict changes in the system.

For a smooth, zero-mean time series, it can be used autoregressive moving average model ARMA (n, m) be fitted:

$$
x_{t}-\varphi_{1} x_{t-1}-\varphi_{2} x_{t-2}-\cdots-\varphi_{n} x_{t-n}=\varepsilon_{t}-\theta_{1} \varepsilon_{t-1}-\cdots-\theta_{m} \varepsilon_{t-m}
$$

If we can choose the appropriate coefficients $k: \varphi_{k 1}, \varphi_{k 2}, \cdots, \varphi_{k k}$, representing a linear combination $x_{i} x_{t-i}$, namely:

$$
x_{t}=\sum_{i=1}^{k} \varphi_{k i} x_{t-i}
$$

When the error variance of this representation: $J=E\left[\left(x_{t}-\sum_{i=1}^{k} \varphi_{k i}\right)^{2}\right]$ the very hour, the last one factor $\varphi_{k k}$ is defined as the partial autocorrelation function. The partial derivative $J$ separately and $\varphi_{k i}$ make it equal to zero, were taken $i=1,2, \cdots, k$, taking into account $\rho_{i}=\rho_{-i}$, in the form of a matrix can be obtained as follows:

$$
\left[\begin{array}{cccccc}
\rho_{0} & \rho_{1} & \rho_{2} & \rho_{3} & \cdots & \rho_{k-1} \\
\rho_{1} & \rho_{0} & \rho_{1} & \rho_{2} & \cdots & \rho_{k-2} \\
\vdots & \vdots & \vdots & \vdots & & \vdots \\
\rho_{k-1} & \rho_{k-2} & \rho_{k-3} & \rho_{k-4} & \cdots & \rho_{0}
\end{array}\right]\left[\begin{array}{c}
\varphi_{k 1} \\
\varphi_{k 2} \\
\vdots \\
\varphi_{k k}
\end{array}\right]=\left[\begin{array}{c}
\rho_{1} \\
\rho_{2} \\
\vdots \\
\rho_{k}
\end{array}\right]
$$

Wherein, $\rho_{0}=1$ the above formulais called Yule-Walker equations.

Model. ARMA model parameter estimation process is non-linear regression procedure, required nonlinear least squares method or other methods to estimate parameters, the calculation process is complex and computationally intensive. Nonlinear regression long autoregressive method by solving a system of linear equations, the ARMA model parameter estimation of linear regression problem into treatment, can greatly reduce the complexity of parameter estimation [2].

Steps to establish ARMA model regression method using long since as follows:

(1) Pre-processing, data that is smooth, zero-mean standardization, normalization process. The data which the formula is zero-mean normalized.

(2) Set the order, which determine the value of $n$ and $m$. You can use any one or guidelines to determine the order.

(3) Estimates of the estimated model parameters and.

(4) Test, that test the applicability of the model to sample data. The most fundamental way to test the applicability of the model is to test whether the white noise.

Examples of Calculation. According to the model used for a wind farm in Zhangjiakou 24 hours to predict the wind speed, the results shown below. Where the solid line is the measured data, the dotted line is the forecast data. 


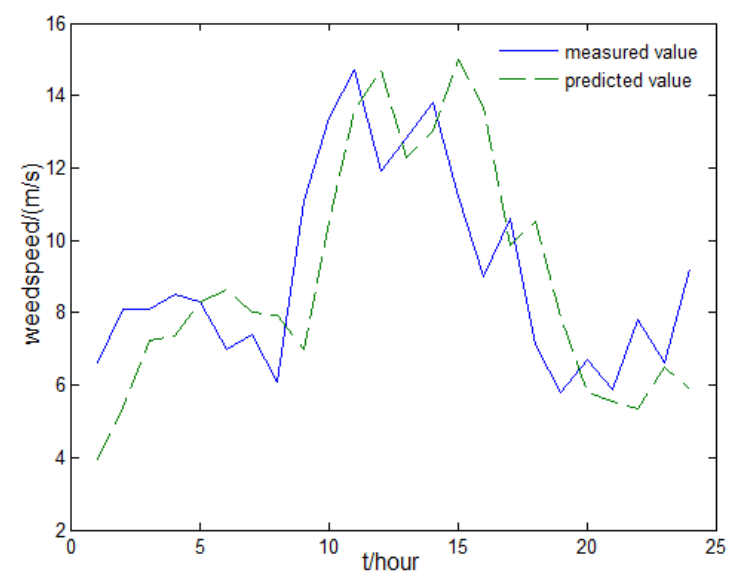

Fig. 1 Time series prediction

As can be seen from the figure, most of the time high prediction accuracy, but to predict the effect of the poor at individual points, especially at the turning point predictions in time series of wind speed was not very accurate. In order to see the prediction accuracy can be calculated for each hour of wind speed prediction relative error relative error calculation formula is:

$$
R E=\frac{(v-\hat{v})}{v} \times 100 \%
$$

The average absolute error is calculated as:

$$
A A E=\frac{1}{N} \sum_{t=1}^{N} \frac{\left|v_{t}-\hat{v_{t}}\right|}{v_{t}} \times 100 \%
$$

Calculated to obtain the absolute average error of the time series model is about $25 \%$, the result was satisfactory. Description time series data point in most able to give a more accurate result, has some practical value, but predicts individual point there are still too large errors.

\section{BP Neural Network Algorithm Network Model was Used to Predicted Wind Speed}

BP Network Model. The neural network has a strong nonlinear fitting capabilities, can be mapped to any complex nonlinear relationship, and learning rules are simple, easy to implement computer. Has strong robustness, memory capacity and a strong self-learning ability, can be used in many occasions. Neural networks is generally a non-linear components multi-input / single-output, its structural model shown in Figure 2.

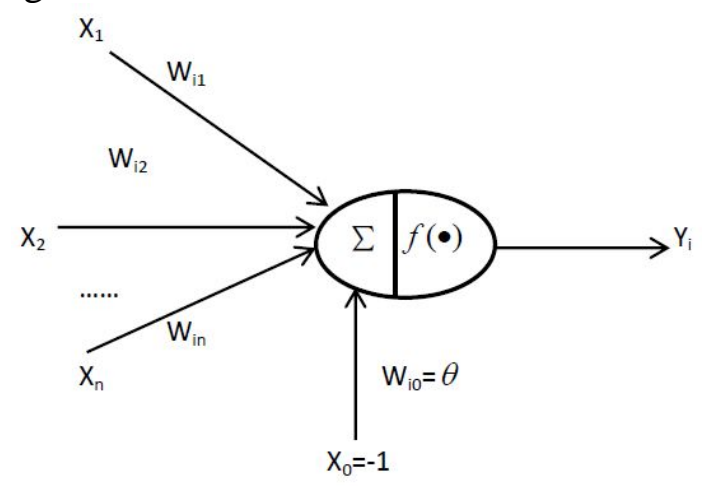

Fig. 2 Artificial neuron model

BP network is one of the most widely used feed-forward neural network, adjusting the rules its name from the network weights uses back-propagation learning algorithm, Back-propagation (BP) learning algorithm. It includes an input layer, one or more hidden layer neurons and an output layer, layer one-way connection between the inner layer neurons are independent of each other [3]. One important feature of the neural network is learning, only users with learning ability, the neural network to have intelligence. 
In the training set size in certain circumstances, the generalization capability of the network directly related to the size of the network. Regularization method is the generalization ability of the network to enhance the performance of the function by adjusting the network. Common BP networks use rms of the network and as a performance function, such as the formula (7):

$$
E=m s e=\frac{1}{N} \sum_{i=1}^{N} e_{i}^{2}=\frac{1}{N} \sum_{i=1}^{N}\left(t_{i}-y_{i}\right)^{2}
$$

Which $e_{i}, t_{i}, y_{i}$ represent the first training samples of training error $i$, the target output and network output. The network performance as a function of the adjusted formula (8) below:

$$
\text { msereg }=r m e s+(1-r) m s w
$$

You can use this function to ensure that the performance of the network training error as small as possible under the circumstances of having a smaller effective network weights and thresholds, which in fact is equivalent to automatically reduce the size of the network, so that the output of the network of training more smooth, thus enhancing network generalization capability [4].

Examples of Calculation. Winds from the sequence predicted time series method is still used in the original data to be solved, finally get BP neural network as shown below:

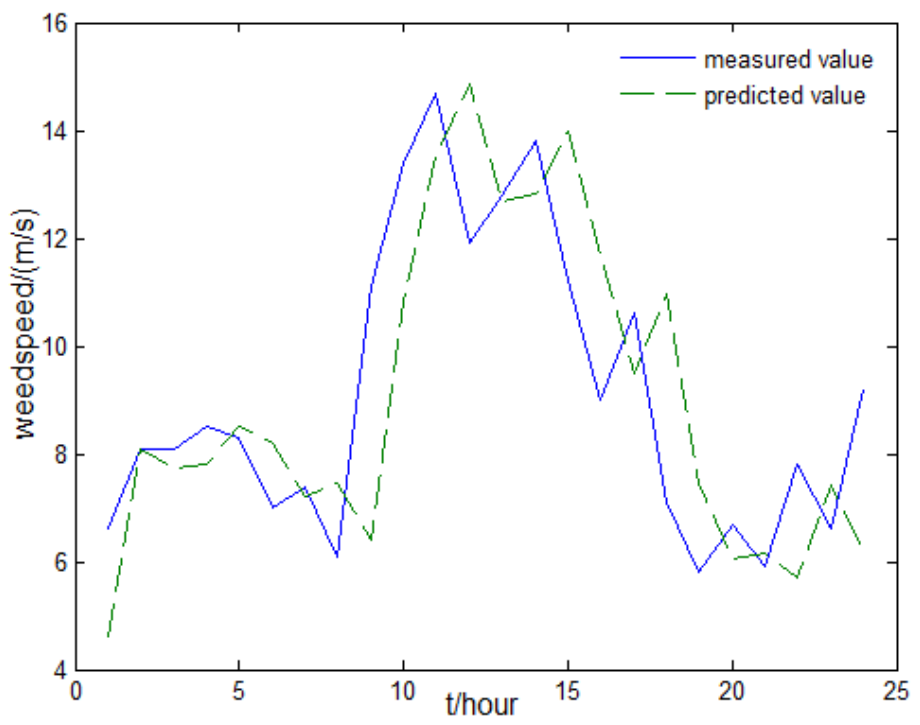

Fig. 3 BP neural network prediction

In fact the same input variable time series and neural network method, but neural network can learn the nonlinear law wind speed, so in theory, neural network algorithm should have a better prediction. After calculation, prediction error BP neural network algorithm is about $22 \%$, showing that it is higher than the prediction accuracy of neural sequences law, in practice, have more practical.

\section{Multi-combination Forecasting Model}

The Concept of Multi-Model Combination Forecasting. As can be seen from the first two, time series method and BP neural network in predicting wind speed there were some errors, in order to further reduce the error, we propose a multi-model combination forecasting method. Multi-combination forecasting model considering the characteristics of each individual prediction methods, different combinations of individual prediction methods, and in an appropriate form obtained a weighted average combination forecasting model.

In accordance with the different combinations of weights, combination forecasting method can be divided into fixed and variable weight coefficient combination method weight coefficient combination method [5]. Currently used to determine the weights of the various methods are assumed to determine the value of the weights, but in practice different characteristics of different forecasting methods, and each method when the prediction accuracy is high low, if possible with the individual weighting coefficient method Accuracy change, then the resulting composition 
constructed to predict the effect of the model will be greatly improved, thus establishing variable weight combination forecasting model has some practical significance. This combination forecast model described below.

Set $x(t)(t=1,2, \cdots, n)$ a prediction target actual value of the first time in, $p$ is a different forecast model predictions at the time $\hat{x_{1}}(t), \hat{x_{2}}(t), \cdots, \hat{x_{p}}(t)$, respectively, then the result is a linear combination of prediction:

$$
\hat{x}(t)=l_{1} \hat{x}_{1}(t)+l_{2} \hat{x}_{2}(t)+\cdots+l_{p} \hat{x}_{p}(t)
$$

Where is a $\mathbf{L}=\left(l_{1}, l_{2}, \cdots l_{p}\right)^{\mathrm{T}}$ weighting coefficient and satisfies

$$
l_{1}+l_{2}+\cdots+l_{p}=1
$$

The first models in the prediction error for the first time

$$
e_{i t}=x(t)-\hat{x}_{i}(t), i=1,2, \cdots, p ; t=1,2, \cdots, n
$$

Corresponding prediction error information matrix:

$$
\mathbf{E}=\left[\left(e_{i t}\right)_{p \times n}\right]\left[\left(e_{i t}\right)_{p \times n}\right]^{\mathrm{T}}
$$

The linear combination of prediction error sum of squares is

$$
\mathbf{J}=\sum_{t=1}^{n}[x(t)-\hat{x}(t)]^{2}=\sum_{t=1}^{n}\left(\sum_{i=1}^{p} l_{i} e_{i t}\right)^{2}=\mathbf{L}^{\mathrm{T}} \mathbf{E} \mathbf{L}
$$

In the prediction error sum of squares minimum objective function [22], to select the right combination of value by solving the equation (14):

$$
\left\{\begin{array}{l}
\min \mathbf{J}=\mathbf{L}^{\mathrm{T}} \mathbf{E} \mathbf{L} \\
\text { s.t. } \mathbf{R}^{\mathrm{T}} \mathbf{L}=1, \quad \mathbf{R}=\left(1,1, \cdots, 1^{\mathrm{T}}\right.
\end{array}\right.
$$

This linear programming problem was solvable weighting coefficients, namely a linear combination forecasting model of the optimal weights is optimal QP model (equation (14)) of.

Calculation Example. In the calculation process, we will time series and neural networks considering two models, a combination of multi-model prediction, and solving the weights obtained at different times of the two models, and ultimately make more models to predict the effect of a combination chart:



Fig. 4 Multi-model prediction of combined

A combination of multi-model means absolute prediction error of about $20 \%$. Clearly, because of a combination of multi-model prediction took into account the different kinds of prediction methods provided information, the prediction error decreases to some extent. So we can conclude: time series and has higher prediction accuracy of neural network model combination forecasting method compared to. 


\section{Wind power prediction}

There are three models already on short-term wind speed plants were predicted and achieved certain result. The next question will highlight the prediction of wind power generation. As the first section of the article, determine the status of wind speed wind turbine power generation size and power status, so it can be relationship and wind power generation units with a wind turbine between the power curve described. Figure 5 is a standard model of wind turbine power curve. Its cut-in speed $\left(v_{\text {in }}\right)$, cut-out wind speed $\left(v_{r}\right)$ and rated speed $\left(v_{\text {out }}\right)$, respectively $4 \mathrm{~m} / \mathrm{s}, 25 \mathrm{~m} / \mathrm{s}$ and $15 \mathrm{~m} / \mathrm{s}$, the rated active power output of the wind turbine.

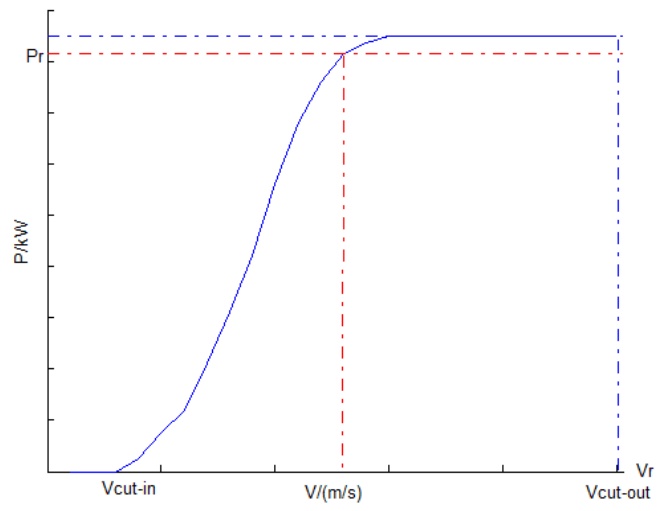

Fig. 5 Wind turbine power curve standard

To make it easier to calculate the wind speed corresponding wind power, wind power generation can use the following characteristics to approximate piecewise functions:

$$
P= \begin{cases}0 & v<v_{\text {in }} \text { or } v>v_{\text {out }} \\ \frac{v-v_{\text {in }}}{v_{r}-v_{\text {in }}} P_{r} & v_{\text {in }}<v<v_{r} \\ P_{r} & v_{r}<v<v_{\text {out }}\end{cases}
$$

Approximately the cut-in speed (15) to cut out the power characteristics of wind speed between the curve is represented by a linear model, the theory is possible.

The following are using time series method, BP neural network and multi-model combination method for wind turbine power predictable results:

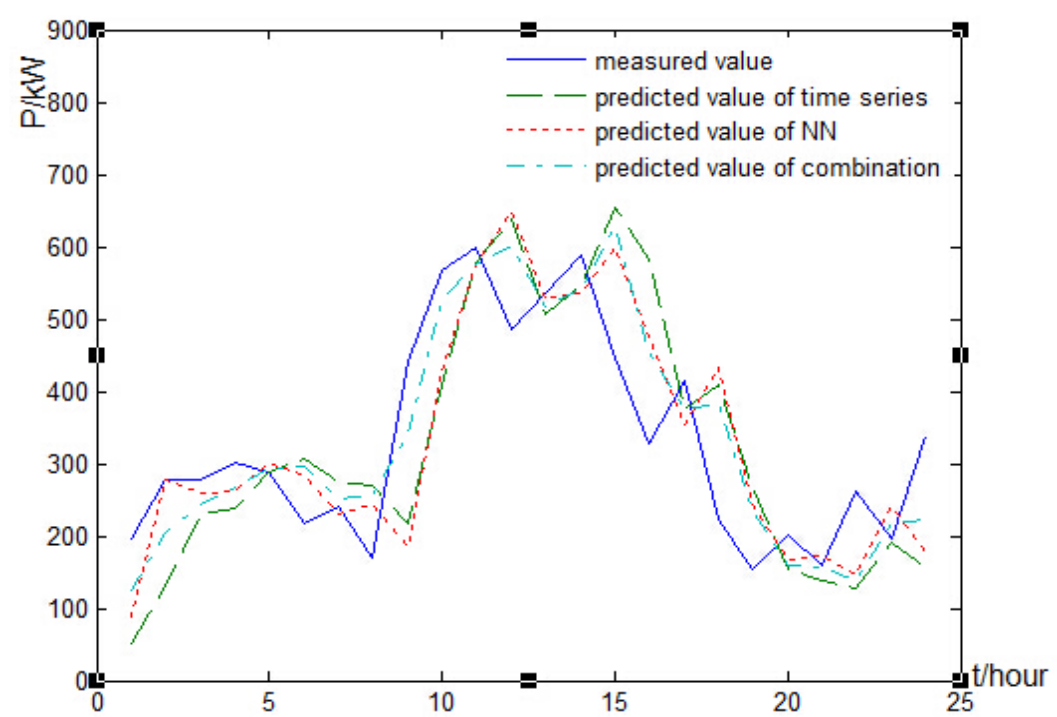

Fig. 6 Wind power forecast map 
Table 1 Comparison of average absolute error of wind speed and wind power prediction

\begin{tabular}{c|c|c}
\hline Prediction target & Wind speed & Wind power generation \\
\hline Prediction method & $24.87 \%$ & $35.06 \%$ \\
\hline Neural network & $21.64 \%$ & $28,12 \%$ \\
\hline Multi-model combination & $19.92 \%$ & $24.06 \%$ \\
\hline
\end{tabular}

From Table 1 can be found, the wind power prediction error of the prediction error is greater than the wind speed. This is because the wind speed does not have a strong regularity, and wind power is obtained by converting the power curve, during the conversion process further weakened its regularity, such that the prediction error becomes large. Therefore, in order to improve the prediction accuracy, we often choose to predict the wind speed.

\section{Conclusion}

Short-term wind power prediction is important for power system safe and stable operation and improvement of operational efficiency. This paper uses neural sequence method, neural network and multi-model combinations of variable weights method to predict winds. Compared with various methods, this paper finds a combination of multi-model prediction method has higher prediction accuracy. Based on the wind speed prediction data and converses the wind speed data to rate data, obtained power prediction curve, and wind speed and wind power prediction errors in a comparative study found that wind power prediction error is greater than the wind speed prediction error.

Although the wind speed and wind farm power forecasting is difficult, the forecast did not reach the limit, through continuous improvement and perfection, the prediction accuracy can be further improved [6].

\section{References}

[1] J. Li, H. Song, W.S. Wang. Large VSCF Wind Turbine Modeling and Simulation. Chinese CSEE, 2004,06: 104-109.

[2] S.Z. Yang, Y. Wu, J.P. Xuan. Time Series Analysis Engineering Applications. Wuhan: Huazhong University of Science and Technology Press, 2007.

[3] Y.X. Yuan, X. Yang. Proceedings Wind Farm Wind Speed and Power Output Forecasting. Chinese CSEE, 2005, 11: 1-5.

[4] Y.D. Xiao. Mountain Wind Farm Capacity to Predict Short-Term Study. Xinjiang University, 2007.

[5] Y.J. Zhang. Variant Weight Combination Forecasting Model in Power System Load Forecasting. Southwest Jiaotong University, 2007.

[6] X. Yang. Wind Farm Wind Speed and Power Output Prediction. Tohoku Electric Power University, 2005. 